\title{
EEG - Controlled Wheelchair Movement: Using Wireless Network
}

\section{Pranob Kumar Charles, Murali Krishna*, Praneeth Kumar GV and Lakshmi Prasad D}

Electronics and Communication Department, Andhra Loyola Institute of Engineering and Technology, JNTUK, Vijayawada, Andhra Pradesh, India

\begin{abstract}
This project discusses about a brain controlled wheel chair based on Brain-computer interfaces (BCl). BCl's are systems that can bypass conventional channels of communication (i.e., muscles and thoughts) to provide direct communication and control between the human brain and physical devices by translating different patterns of brain activity into commands in real time. The intention of the project work is to develop a robot that can assist the disabled people in their daily life to do some work independent of others.

Here, we analyze the brain wave signals. Human brain consists of millions of interconnected neurons, the pattern of interaction between these neurons are represented as thoughts and emotional states. According to the human thoughts, this pattern will be changing which in turn produce different electrical waves. A muscle contraction will also be generate a unique electrical signal. All this electrical waves will be sensed by the brain wave sensor it will convert the data into packets and transmit through Bluetooth medium. Level analyzer unit (LAU) will receive the brain wave raw data and it will extract and process the signal using MATLAB platform. Then the control commands will be transmitted to the robot module to process. With this entire system, we can move a robot according to the human thoughts and it can be turned by blink muscle contraction.
\end{abstract}

Keywords: Brain-computer interfaces (BCI); Level analyzer unit (LAU); Blink muscle contraction

\section{Introduction}

Brainwaves are the crucial of this system. There are various kinds of waves based on our Meditation and Attention levels. We set some threshold values, which guide the wheelchair for forward or backward movement and steering. The wheelchair is properly calibrated when it's not used to ensure that it functions optimally [1]. The Wheelchair is integrated with ultrasonic sensor. It is used to detect the distance between obstacle and wheelchair [2]. If the obstacle is very nearer to wheelchair, the wheelchair moves backward. Next, the meditation and attention levels of the patient are recorded with a brainwave sensor, which also transmits them wirelessly to the PC by using Bluetooth. Subsequently, these waves are sent to Arduino module by using Zigbee module. If the patient blinks both the eyes, the wheelchair is turned left or right, depending on the number of blinks. As a result, the patients are made to be autonomous and self-reliant [3-5].

\section{Block Diagram Description}

Firstly, a dry electrode is used to record the brain waves. These raw waves are sent via Bluetooth to a data processing unit whose output is serial data. Subsequently, this data is sent to Arduino. Furthermore, the Arduino is connected to a display and two motors which rotate according to the commands of Arduino [6-10].

Here, the data processing unit is programmed using IDE such as Matlab and interfaced with Arduino. Moreover, Arduino is also programmed by using an Arduino IDE [11]. Furthermore, Thinkgear Connector scans the ports for EEG signal and sends it to Matlab for further processing (Figure 1).

\section{Flow Chart}

Initially, Bluetooth should be turned on. Next, the brain wave sensor acquires the EEG signals from the brain using a dry electrode. Subsequently, these values are transmitted to Matlab using Bluetooth. Now, these values are sent to protype using Zigbee connection (Figure 2).

\section{Existing System}

In the existing system, patient must depend on other person for moving wheelchair. It is more time consuming task and it is less effective [12]. Moreover, the normal person may not be available all the time.

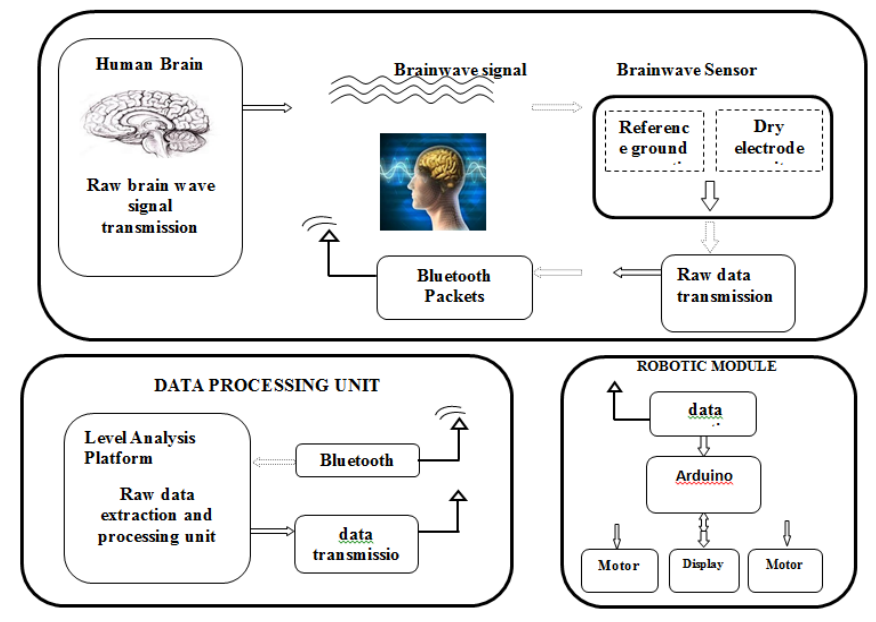

Figure 1: Block Diagram .

*Corresponding author: Murali Krishna, Electronics and Communication Department, Andhra Loyola Institute of Engineering and Technology, JNTUK, Vijayawada, Andhra Pradesh, India, Tel: 0866249 8978; E-mail: murali.krishna526@gmail.com

Received March 02, 2018; Accepted March 20, 2018; Published March 27, 2018

Citation: Charles PK, Krishna M, Kumar GVP, Prasad DL (2018) EEG - Controlled Wheelchair Movement: Using Wireless Network. J Biosens Bioelectron 9: 252. doi:10.4172/2155-6210.1000252

Copyright: (c) 2018 Charles PK, et al. This is an open-access article distributed under the terms of the Creative Commons Attribution License, which permits unrestricted use, distribution, and reproduction in any medium, provided the original author and source are credited. 
As a result, it becomes uncomfortable for both patient and his/her helper. Furthermore, pushing a wheelchair is a mundane task and it requires lots of concentration. In the end, the hospital may be crowded due to increased number of visitors $[13,14]$.

\section{Proposed System}

In this system, the disabled people needn't call others to move their wheelchair. Instead, a robotic module that consist of various components like Arduino, Brainwave Sensor, and Zig-bee will take care of the task. In other words, the nerves of the patient are bypassed using this wireless technology [15]. An Ultrasonic sensor is used, which detects obstacles and moves the wheelchair backwards. The steering of the wheelchair is guided by using eye blink of the patient $[16,17]$.

\section{Methodology}

\section{Ultrasonic sensor}

The sensor is used to detect the level of the dust in the dustbin. It uses a sound transmitter and receiver. An ultrasonic sensor create an ultrasonic pulse called ping and listen for the reflection of pulse. The sound pulse is created electronically using a sonar projector consisting of signal generator, power amplifier, and electro-acoustic transducer array. A beam former is usually employed to concentrate the acoustic power into the beam.

It measures the distance of an object by using sound waves. It sends out a sound wave at a specific frequency and listening for that sound wave to bounce back (Figure 3).

\section{Arduino Uno}

Arduino Uno is a simple microcontroller which is used in making interactive projects like drone, EEG-based wheelchair, and so on. A program that is almost nearer to $\mathrm{C}++$ is written on the Arduino IDE. Next, it is dumped into the Arduino. However, we can erase the program on the Arduino by pressing reset button. It has 14 digital input/output pins (of which 6 can be used as PWM outputs), 6 analog inputs, a 16 $\mathrm{MHz}$ quartz crystal, a USB connection, a power jack, an ICSP header, and a reset button (Figure 4).

\section{Mindwave sensor}

Mindwave sensor for Arduino can record the EEG waves of the mind.

It has a dry electrode, an ear clip, and a Bluetooth transmitter, which are combined together to transmit brain waves (Figure 5).

\section{Zig-bee module}

Zigbee is an IEEE 802.15.4-based specification for a suite of high-level communication protocols used to create personal area networks with small, low-power digital radios, such as for home automation, medical device data collection, and other low-power lowbandwidth needs, designed for small scale projects which need wireless connection. Hence, Zigbee is a low-power, low data rate, and close proximity (i.e., personal area) wireless ad hoc network (Figure 6).

The technology defined by the Zigbee specification is intended to be simpler and less expensive than other wireless personal area networks (WPANs), such as Bluetooth or more general wireless networking such as Wi-Fi. Applications include wireless light switches, home energy monitors, traffic management systems, and other consumer and industrial equipment that require short-range lowrate wireless data transfer (Figure 7).

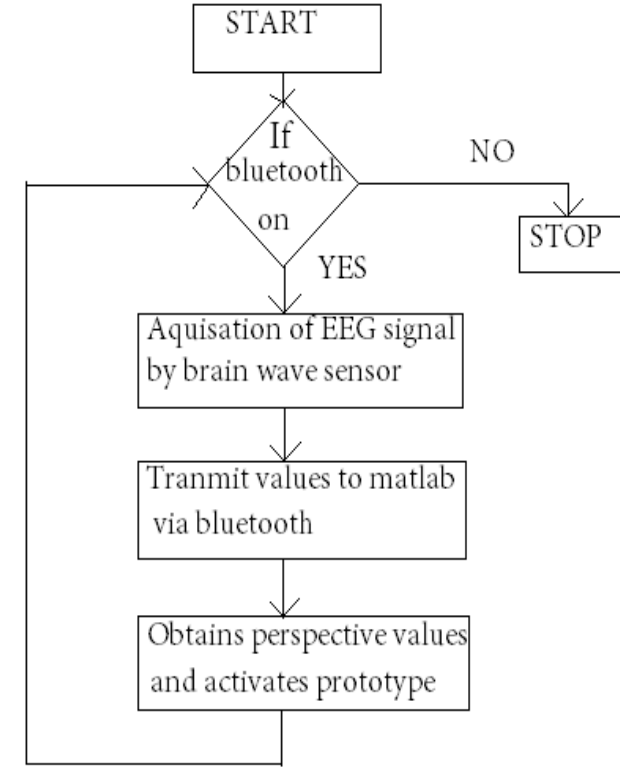

Figure 2: Flow chart

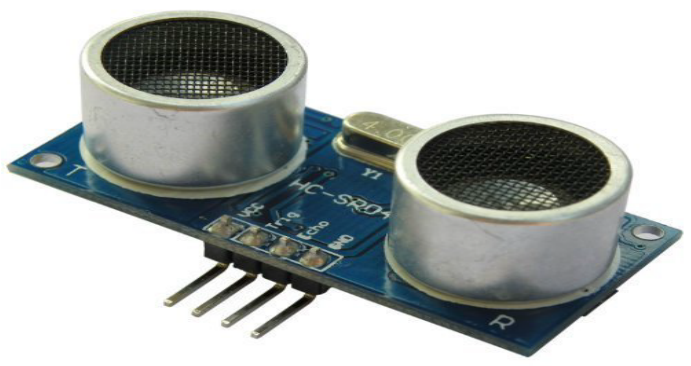

Figure 3: Ultrasonic sensor

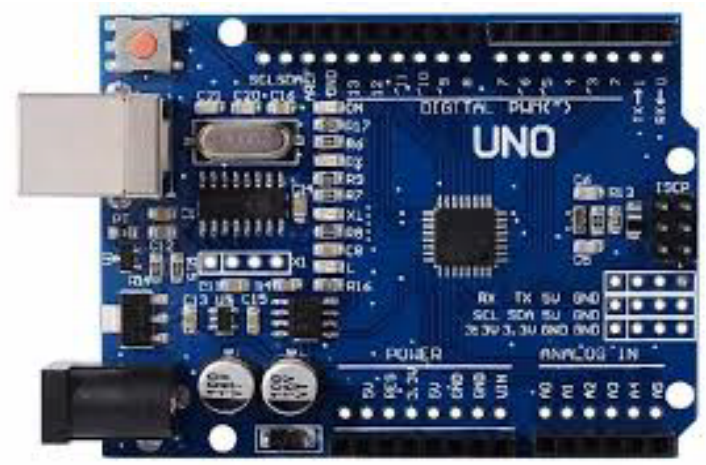

Figure 4: Arduino Atmega 328p.

\section{Results and Discussion}

The following are the results which obtained from this work

- Wheel chair can be moved just by using brainwaves

- Transmit the information wirelessly to motors 
Citation: Charles PK, Krishna M, Kumar GVP, Prasad DL (2018) EEG - Controlled Wheelchair Movement: Using Wireless Network. J Biosens Bioelectron 9: 252. doi:10.4172/2155-6210.1000252

- The data of the brain waves can be stored and it is retrieved later.

- The real-time data transmission and access

- Obstacles are taken care of by using Ultrasonic sensor.

- Avoids dependence on others.

This EEG based wheel chair is very useful for disabled patients in different aspects. We have seen that, our hand muscles are not involved in moving the prototype (Figure 8).

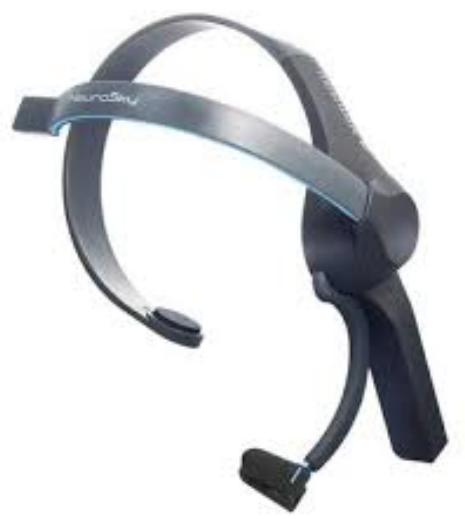

Figure 5: Mindwave sensor.

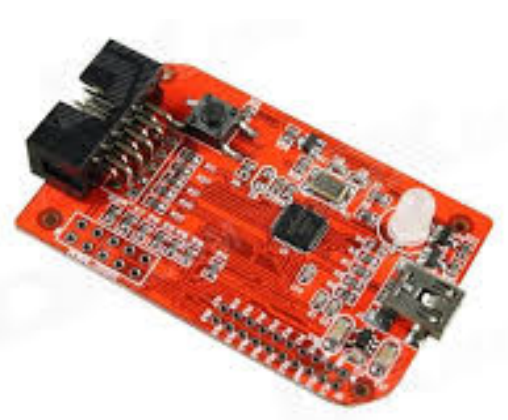

Figure 6: zig-bee module.

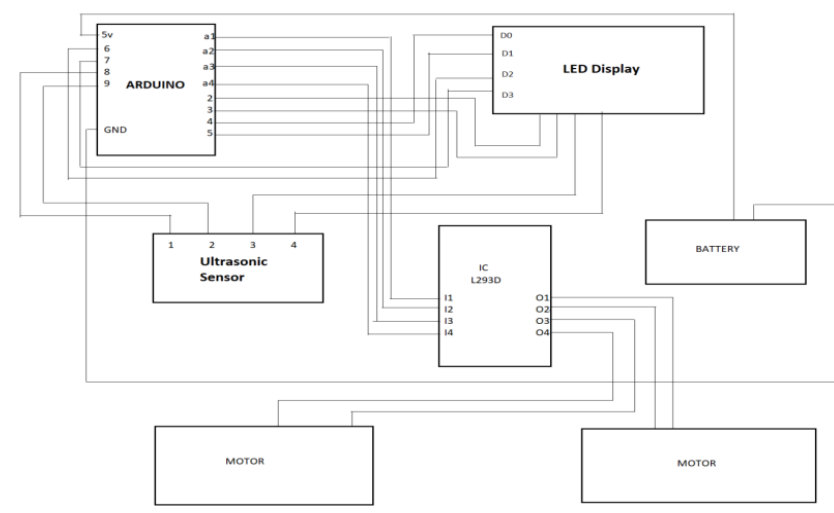

Figure 7: circuit diagram

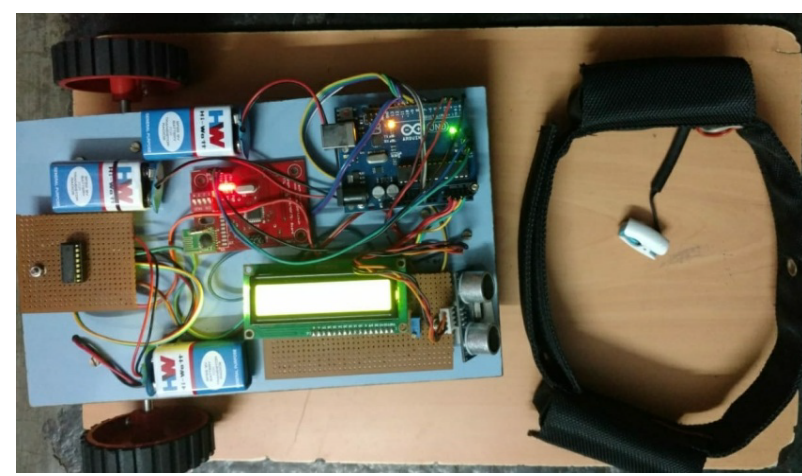

Figure 8: EEG based wheel chair

\section{Conclusion}

The objective of the project is for the real time support for disabled and paralysed patients. Once implemented, we are sure that it is an invaluable tool for both patients and doctors. In this information about the brainwaves is sent and movement is performed immediately based on their values. The range is very small, as the patient is very near to the wheelchair. The attention and meditation values vary for different kinds of persons. However, they are normally low for disabled people. Hence, the threshold values are set very low. Moreover, the doctors easily get the information about the patient's brain waves using this setup.

\section{Future Work}

We can add some complementary features like Artificial intelligence, Cruise control, distress call, and an alarm. However, the basic functionality remains unchanged except for blind people, for whom we insert the electrode in the brain.

\section{Acknowledgment}

We are technically supported by our project guide. We were encouraged by our head of the department and our department.

\section{References}

1. Gao S, Wang Y, Gao X, Hong B (2014) Visual and auditory brain-computer interfaces. IEEE Trans Biomed Eng 61: 1436-1447.

2. Hammon PS, Virginia R (2007) Preprocessing and meta-classification for braincomputer interfaces. EEE Trans Biomed Eng 54: 518-525.

3. Schalk G, McFarland DJ, Hinterberger T, Birbaumer N, Wolpaw JR (2004) $\mathrm{BCl}$ 2000: A general-purpose brain-computer interface $(\mathrm{BCl})$ system. EEE Trans Biomed Eng 51:1034-1043.

4. Moore MM (2003) Real-world applications for brain-computer interface technology. IEEE Trans Neural Syst Rehabil Eng 11: 162-165.

5. Schalk G, Eric CL (2011) Brain-computer interfaces using electrocorticographic signals. IEEE Rev Biomed Eng 4:140-154.

6. Long J, Li Y, Wang H, Yu T, Pan J, et al. (2012) A hybrid brain computer interface to control the direction and speed of a simulated or real wheelchair. IEEE Trans Neural Syst Rehabil Eng 20: 720-729.

7. Marshall D, Coyle D, Wilson S, Callaghan M (2013) Games gameplay and BCl: the state of the art. IEEE T Comp AI 5: 88-99.

8. Guger C, Ramoser H, Pfurtscheller G (2000) Real-time EEG analysis with subject-specific spatial patterns for a brain-computer interface $(\mathrm{BCI})$. IEEE Trans Rehabil Eng 8: 447-456.

9. Maeder CL, Sannelli C, Haufe S, Blankertz B (2012) Pre-stimulus sensorimotor 
Citation: Charles PK, Krishna M, Kumar GVP, Prasad DL (2018) EEG - Controlled Wheelchair Movement: Using Wireless Network. J Biosens Bioelectron 9: 252. doi:10.4172/2155-6210.1000252

rhythms influence brain computer interface classification performance. IEEE Trans Neural Syst Rehabil Eng.

10. Makeig S, Kothe C, Mullen T, Bigdely-Shamlo N, Zhang Z, et al. (2012) Electron spectroscopy studies on magneto-optical media and plastic substrate interface. Proceedings of the IEEE.

11. Alexander M, Dan Z, Yijun W, Shangkai G, Andreas KE (2011) Multimodal brain-computer interfaces. Tsinghua Sci Technol 16: 133-139.

12. Mak JN, Wolpaw JR (2009) Clinical applications of brain computer interfaces: current state and future prospects. IEEE Rev Biomed Eng 2: 187-199.

13. Yuan H, He B (2014) Brain-computer interfaces using sensorimotor rhythms current state and future perspectives. IEEE Trans Biomed Eng 61: 1425-1435.
14. Wang Y, Gao X, Hong B, Jia C, Gao S (2008) Brain computer interfaces based on visual evoked potentials. IEEE Engineering in Medicine And Biology Magazine.

15. Murat A, Betts P, Moghadamfalahi M, Mooney AR, Orhan U, et al. (2014) Noninvasive brain-computer interfaces for augmentative and alternative communication. IEEE Rev Biomed Eng 7: 31-49.

16. Frisoli A, Loconsole C, Leonardis D, Bann'o F, Barsotti M, et al. (2012) A new gaze-BCl-driven control of an upper limb exoskeleton for rehabilitation in realworld tasks. IEEE Trans Syst Man Cybern 42: 1169-1179.

17. Hill NJ, Thomas NL, Schröder M, Hinterberger T,Wilhelm B, et al. (2006) Classifying EEG and ECoG signals without subject training for fast $B C$ implementation: comparison of nonparalyzed and completely paralyzed subjects. IEEE Trans Neural Syst Rehabil Eng 14: 183-186. 